\title{
EL COMPLEJO DE CAMPYLONEURUM ANGUSTIFOLIUM (SW.) FÉE (POLYPODIACEAE) EN COSTA RICA
}

\author{
Alexánder Fco. Rojas Alvarado \\ Jardín Botánico Lankester, Universidad de Costa Rica, apdo. 1031-7050 Cartago, Costa Rica. \\ afrojasa@hotmail.com
}

\begin{abstract}
The Campyloneurum angustifolium complex in Costa Rica is analyzed. As a result, a new species is described (C. gracile A.Rojas), a comparative table and a key are included for the group.
\end{abstract}

Resumen. Se analiza el complejo de Campyloneurum angustifolium en Costa Rica. Como resultado, se describe una nueva especie (C. gracile A.Rojas) y se ofrece un cuadro comparativo y una clave para el grupo.

Palabras Clave / Key Words: Pteridophyta, Polypodiaceae, Campyloneurum, Costa Rica.

En el complejo de Campyloneurum angustifolium (Sw.) Fée, Lellinger (1988) describe C . centrobrasilianum, $C$. cooperi y $C$. irregulare como especies nuevas y realiza la combinación $C$. densifolium (Hieron.) Lellinger. Este autor compara la primera especie con C. angustipaleatum (Alston) M. Mey. ex Lellinger, la segunda con $C$. rigidum $\mathrm{J}$. Sm. y la tercera con $C$. amphostenon (Kunze ex Klotzsch) Fée.

Lellinger(1989) indica la presencia de Campyloneurum cooperi y C. irregulare en Costa Rica; sin embargo, León (1995) sinonimiza estos nombres con C. angustifolium s.l. y argumenta que este grupo requiere de mayores estudios. Adicionalmente, el segundo autor registra $C$. angustipaleatum y $C$. densifolium en Costa Rica.

En la presente investigación se analiza la morfología de las especies del complejo de Campyloneurum angustifolium presentes en Costa Rica y se describe una especie nueva. Además, se ofrece una clave dicotómica y un cuadro comparativo de las especies del grupo. Se utilizaron distintos tratamientos taxonómicos en Polypodiaceae de Mesoamérica y Sudamérica (Proctor 1985, 1989, Lellinger 1988, 1989, Mickel \& Beitel 1988, León 1993, 1995, Smith 1995, Rojas 1996) y se revisaron las colecciones del Museo Nacional de Costa Rica (CR) y del Instituto Nacional de Biodiversidad (INB).

Campyloneurum amphostenon (Kunze ex Klotzsch) Fée, Mém. Foug. 5: 258. 1852. Polypodium amphostenon Kunze ex Klotzsch, Linnaea 20: 399. 1847. Tipo: Venezuela, Mérida, Moritz $120 b$ (B).

León (1995) menciona que entre 1500 y 2500 m de altitud existen plantas con características intermedias entre esta especie y Campyloneurum angustifolium, que probablemente corresponden a una especie diferente. Sin embargo, no se ha logrado definir si es un híbrido entre $C$. amphostenon y (probablemente) $C$. irregulare Lellinger (por tener células basales de las escamas del rizoma un poco irregulares) o si es una especie válida, por lo que se mantiene aquí como una variante de $C$. amphostenon, que en el Cuadro 2 ha sido indicada como C. amphostenon 2 para resaltar sus diferencias.

En Mesoamérica, la entidad original de C.amphostenon (véase variante 1 del Cuadro 2) se encuentra sólo en Costa Rica y Panamá y pasa a la Cordillera de Los Andes en Sudamérica, en tanto que la variante 2 se extiende al norte hasta México.

Campyloneurum gracile A. Rojas, sp. nova

Tipo: Costa Rica. San José: Tarrazú, San Marcos, Reserva Biológica Río Paraíso, sendero en la fila al O de Río Negro, 9³3’30”N, 8408'10"W, 500 m, 18 mayo 2004, A. Rojas \& M. Hidalgo 5666 (Holotipo: $\mathrm{CR}$, isotipos: $\mathrm{K}, \mathrm{MO}$ ).

A Campyloneuro angustifolio rhizomatis stipitisque gracilibus, rhizomatis squamis brevioribus et distributione altitudinali inferiore absimilis.

Epifita; rizoma de $1.5-2.5 \mathrm{~mm}$ de diámetro, muy corto, reptante (frondas próximas), negro al secar, no pruinoso; escamas del rizoma de 1.5-3 (-4) x 0.5-1 $\mathrm{mm}$, lanceoladas, pardo oscuro, peltadas, clatradas, con base cordiforme, células de la base redondeadas 


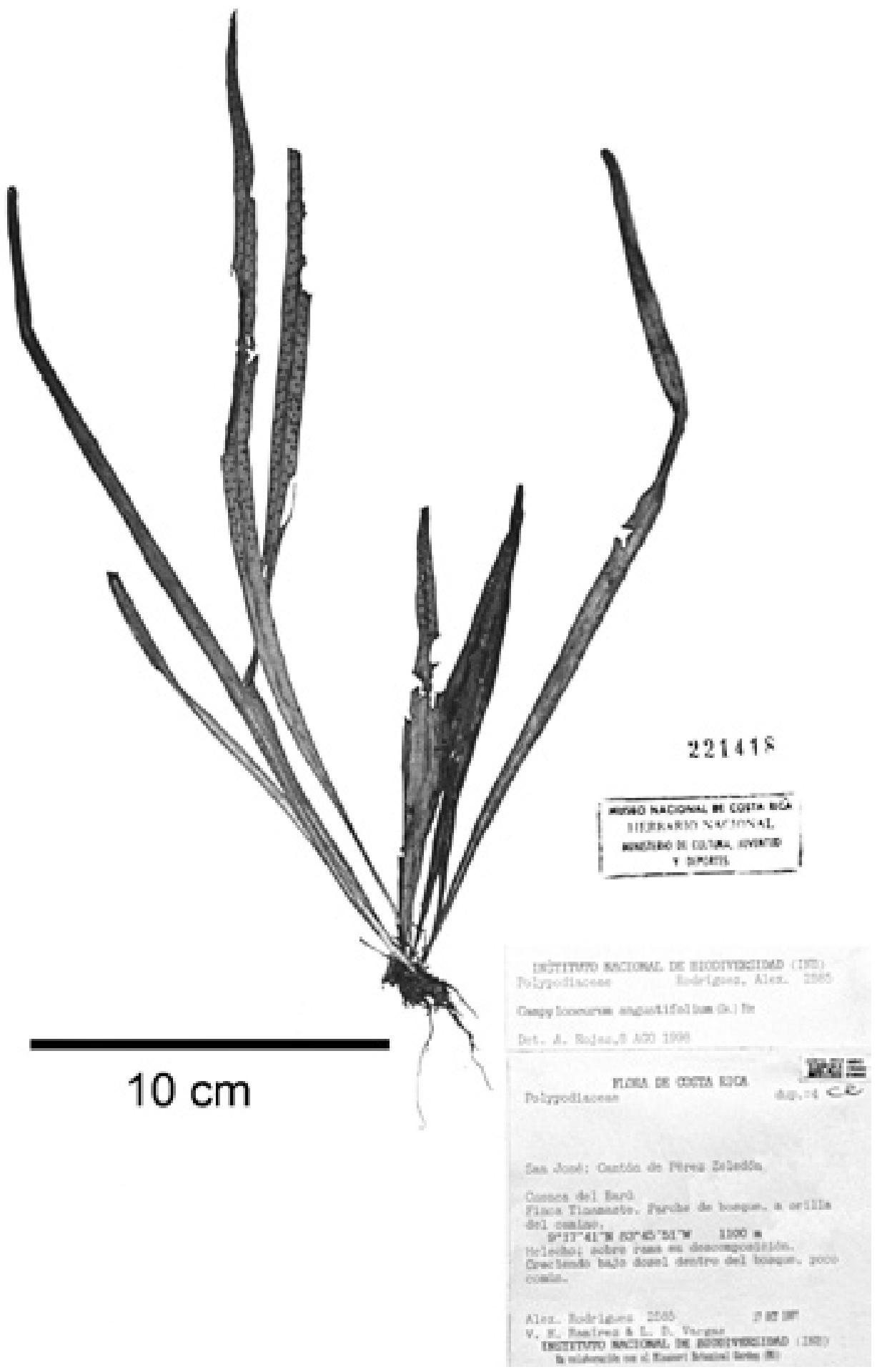

Fig. 1. Ejemplar representativo de Campyloneurum gracile A.Rojas. (A. Rodríguez et al. 2585, CR). 
a poligonales, las del ápice de 1-3 veces más largas que anchas, lumen de las células un poco oscurecido; frondas de (10-) 27-40 (-55) cm de largo, decumbentes; pecíolo de $0.5-4 \mathrm{~cm}$ de largo, 1/20-1/10 del tamaño de la fronda, $0.7-1 \mathrm{~mm}$ de diámetro, pajizo a pardo claro; lámina de (9-) 15-35 (-50) x 0.3-1.5 (-2) cm, linear-elíptica a linear, atenuada en ambos extremos, papirácea a subcartácea, glabra; raquis pajizo a pardo claro, glabro; nervaduras no evidentes; soros redondos, en 1-2 (-3) series irregulares entre la costa y el margen.

DisTRIBUCIÓN. En llanuras, serranías y pies de cordilleras de Costa Rica, Panamá y Colombia, 0-900 (-1200) m.

Paratipos: Costa Rica. Alajuela: Upala, Dos Ríos, 5 $\mathrm{km}$ al S de Brasilia, margen derecha del Río Pizote, $10^{\circ} 55^{\prime} \mathrm{N}, 85^{\circ} 20^{\prime} \mathrm{W}, 500 \mathrm{~m}, 28$ oct 1987 , G. Herrera 955A (CR, INB). Guanacaste: Parque Nacional Rincón de La Vieja, Hacienda Santa María, de la planta hidroeléctrica $0.5 \mathrm{~km}$ sobre el canal subiendo hacia el $\mathrm{O}$ en el primer "puente" de dicho canal, $10^{\circ} 48^{\prime} \mathrm{N}$, $85^{\circ} 19^{\prime} \mathrm{W}, 750-850 \mathrm{~m}, 17$ ago 1987 , G. Herrera 728 (CR, INB, MO). Limón: Talamanca, trail $500 \mathrm{~m} \mathrm{~N}$ of the camp by the River Lari, $9^{\circ} 26^{\prime} 10^{\prime \prime} \mathrm{N}, 83^{\circ} 03^{\prime} 00^{\prime} \mathrm{W}$, 350 m, 29 Febr 1992, J. Bittner 1371 (CR, INB).

San José: Pérez Zeledón, Cuenca del Barú, Finca Tinamaste, $9^{\circ} 17^{\prime} 41^{\prime \prime} \mathrm{N}, 83^{\circ} 45^{\prime} 51^{\prime \prime} \mathrm{W}, 1100 \mathrm{~m}, 17$ ago 1997, A. Rodríguez et al. 2585 (CR, INB, MO).

Otros Especímenes Estudiados: Costa Rica. Alajuela: San Ramón, Peñas Blancas, Río Peñas Blancas, Refugio Aleman's, Quebrada Rojas, $10^{\circ} 18^{\prime} \mathrm{N}, 84^{\circ} 45^{\prime} \mathrm{W}, 850$ 900 m, 29 febr 1992, E. Bello 4474 (INB); lower

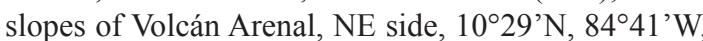
500 m, 17 Sept 1972, R. Lent 2945 (CR); Upala, Bijagua, Finca Zapote, $10^{\circ} 45^{\prime} \mathrm{N}, 85^{\circ} 04^{\prime} \mathrm{W}, 500-530 \mathrm{~m}$, 9 jul 1994, A. Rojas 1260 (INB); Upala, Estación San Cristóbal, Quebrada Cucaracho, $10^{\circ} 52^{\prime} \mathrm{N}, 85^{\circ} 24^{\prime} \mathrm{W}$, 600-620 m, 11 ago 1998, A. Rojas et al. 4744 (CR). Cartago: Turrialba, La Suiza, Chirripó Abajo (BoloriNak), $9^{\circ} 46^{\prime} \mathrm{N}, 8^{\circ} 22^{\prime} \mathrm{W}, 550-700 \mathrm{~m}, 7$ ago $1995, A$. Rojas et al. 2153 (INB). Guanacaste: Santa Cruz, Diriá, camino a la torre, vista del mar, $10^{\circ} 08^{\prime} \mathrm{N}$, $85^{\circ} 37^{\prime} \mathrm{W}, 300-960 \mathrm{~m}, 23$ sept 1996, U. Chavarría 1555 (INB); Parque Nacional Rincón de La Vieja, Hacienda Santa María, siguiendo el canal 1 km sobre el mismo, partiendo de la planta hidroeléctrica, $10^{\circ} 48^{\prime} \mathrm{N}$, $85^{\circ} 19^{\prime} \mathrm{W}, 800-850 \mathrm{~m}, 9$ ago 1987, G. Herrera 662 (CR, INB, MO); ridge above Lago Arenal, $3 \mathrm{~km} \mathrm{~N}$ of Tilarán, 6 Sept 1971, P. Opler 1101 (CR); La CruzSanta Cecilia, Finca La Pazmompa, $11^{\circ} 02^{\prime} 00^{\prime \prime} \mathrm{N}$,
85²4’30" W, 450 m, 9 oct 1990, P. Ríos et al. 99 (CR, INB); La Cruz, Santa Cecilia, Estación Pitilla, Sendero Los Memos, $10^{\circ} 59^{\prime} \mathrm{N}, 85^{\circ} 25^{\prime} \mathrm{W}, 700-800 \mathrm{~m}, 4$ sept 1996, A. Rojas et al. 2985 (INB). Heredia: Sarapiquí, on road between Cariblanco and San Miguel, $10^{\circ} 15$ $16^{\prime} \mathrm{N}, 84^{\circ} 10^{\prime} \mathrm{W}, 700 \mathrm{~m}, 12 \mathrm{Jul}$ 1983, K. Barringer et al. 3738 (CR). Limón: Pococí, Colorado, Sardinas, $10^{\circ} 38^{\prime} \mathrm{N}, 83^{\circ} 34^{\prime} \mathrm{W}, 15-20 \mathrm{~m}, 12$ dic 1992, F. Araya 139 (INB); Talamanca, camp $300 \mathrm{~m}$ up by the river Lari, 9²6’10” N, 8303'00”'W, 320 m, 26 Febr 1992, J. Bittner 1301 (CR, INB); path beyond Río Sucio, Braulio Carrillo, 400-500 m, May 1984, L. Gómez et al. 22751 (CR); Limón, Reserva Indígena Chirripó, Fila de Matama, Raíz de Roble, a orillas de una de las

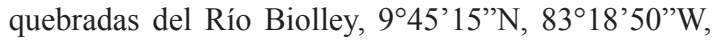
1300-1400 m, 16 ago 1995, A. Rojas et al. 2299 (CR). Puntarenas: Coto Brus, Estación Pittier, Aguas Calientes, ribera del Río Cotón, $9^{\circ} 01^{\prime} \mathrm{N}, 82^{\circ} 57^{\prime} \mathrm{W}$, 1000 m, 31 ene 1995, E. Fletes 50 (INB); San Vito de Java, Finca Wilson, 1500 m, ago 1969, P. León 1181 (CR); San Vito de Java, Las Cruces Botanical Garden, $8^{\circ} 50^{\prime} \mathrm{N}, 82^{\circ} 55^{\prime} \mathrm{W}, 1$ Febr 1988, E. Hennipman et al. 7056 (CR); Guaymí de Coto Brus, 700 m, 19 jun 1977, R. Ocampo 1952 (CR); Jardín Botánico Wilson, Las Cruces, San Vito de Coto Brus, sobre sendero E hacia Río Java, 847’30”N, 8258’00”W, 1100 m, 3 sept 1993, G. Rivera 2150 (CR). San José: Mora, Zona

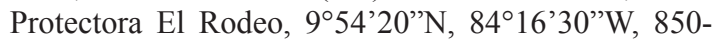
900 m, 5 mayo 1997, A. Cascante 1283 (CR); Santiago de Puriscal, 9 nov 1946, J. Echeverría 821 (CR); Pérez Zeledón, Tinamaste, Finca de los suizos, $9^{\circ} 17^{\prime} 54^{\prime \prime} \mathrm{N}$, 8346’20"W, 700 m, 9 jun 1997, A. Estrada et al. 855 (CR); Turrubares, San Juan de Mata, Cerro Turrubares, $9^{\circ} 49^{\prime} \mathrm{N}, 8^{\circ} 28^{\prime} \mathrm{W}, 1200$ m, 6 dic 1991, R. Zúñiga 589 (INB); Sector San Rafael, Sitio Cerro Pelón. San Luis de Turrubares, 25 jul 1933, M. Valerio 474A (CR); Zona Protectora Cerros de Turrubares, Sector San Rafael, Sitio Cerro Pelón, 949'00”N, 84²8'50”W, 1200 m, 6 dic 1991, R. Zúníga 589 (CR, INB).

PANAMÁ. Colón: hills just N of the Río Guanche, 1200 m, 16 Nov 1975, G. Davidse \& W. D'Arcy 10063 (CR). Darién: Punta Guayabo Grande, along beach and $c a .50 \mathrm{~m}$ from waters edge, 0-50 m, $20 \mathrm{Apr} 1980$, T. Antonio \& W. Hahn 4219 (CR). Panamá: $150 \mathrm{~m}$ de la torre de comunicaciones de INTEL, en dirección al Sendero Panamá, 800-900 m, 13 oct 1994, M. Morris 234 (CR). Veraguas: along trail to summit of Cerro Tute, $c a .3 \mathrm{~km}$ above Escuela de Agricultura Alto Piedra, near Santa Fé, 2600-2800 ft. [790-855 m], 4 Jun 1981, K. Sytsma \& T. Antonio 3008 (CR).

COLOMBIA. Meta: $20 \mathrm{~km}$ SE of Villavicencio, 480 m, 17 Mar 1939, A. Alston 7558 (CR). 

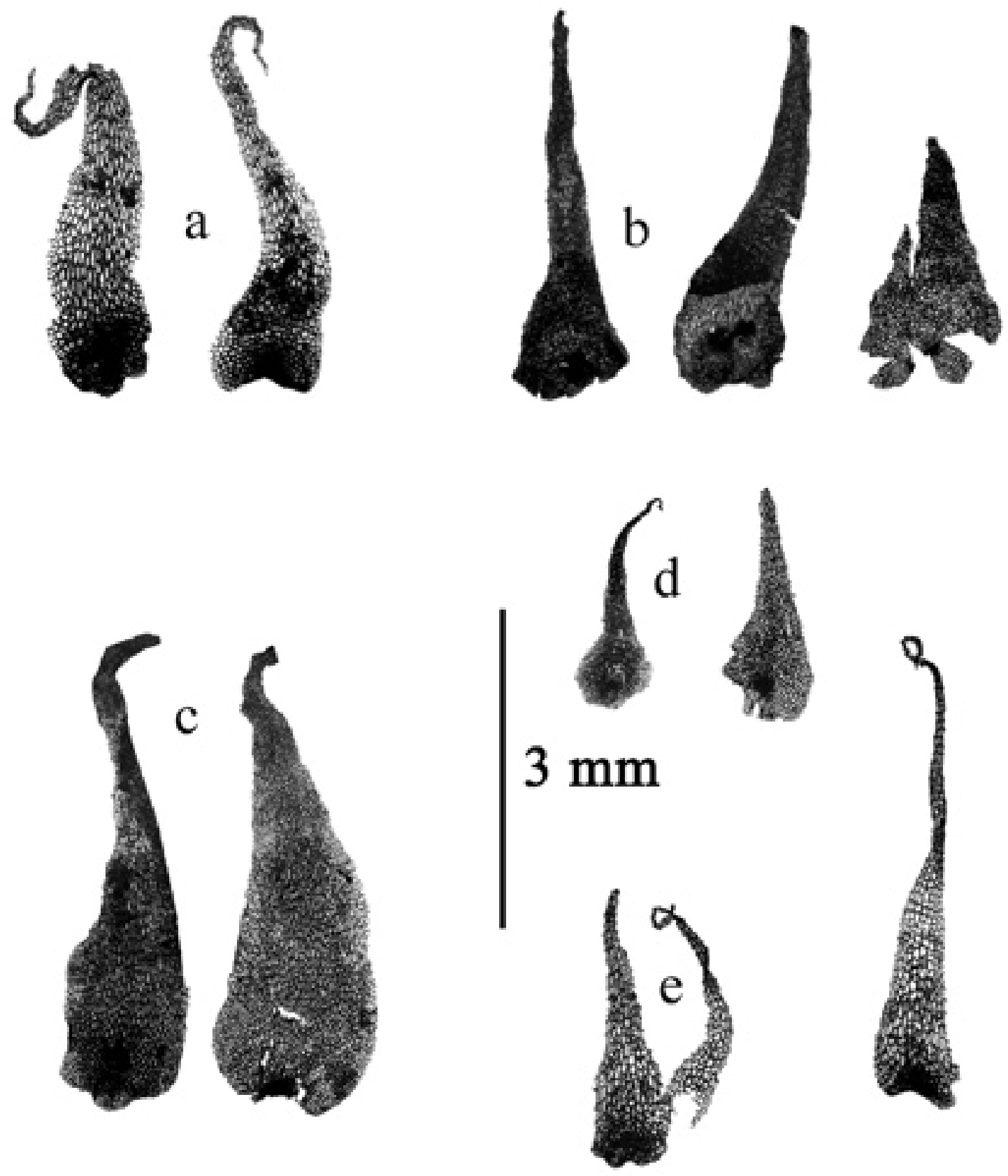

Fig. 2. Escamas del rizoma de: a. Campyloneurum angustifolium (Sw.) Fée. b. C. irregulare Lellinger. c. C. cooperi Lellinger. d. C. gracile A.Rojas. e. C. angustipaleatum (Alston) M.Mey. ex Lellinger. 

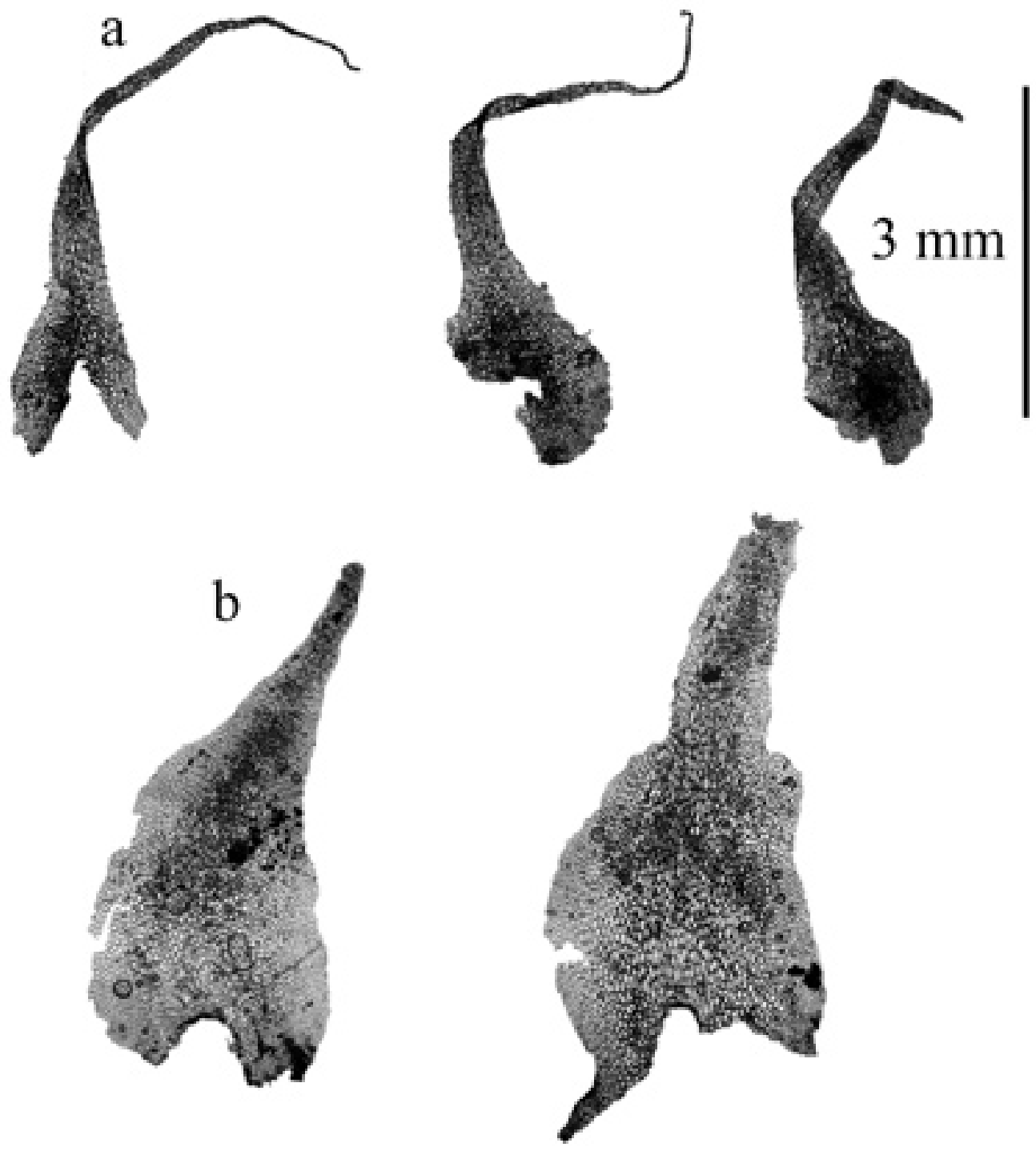

Fig. 3. Escamas del rizoma de: a. Campyloneurum amphostenon (Kunze ex Klotzsch) Fée. b. C. densifolium (Hieron.) Lellinger.

Difiere de Campyloneurum angustifolium (Sw.) Fée por rizoma de 1.5-2.5 $\mathrm{mm}$ de diámetro (vs. 2-5 $\mathrm{mm}$ de diámetro) y no pruinoso (vs. comúnmente pruinoso), escamas del rizoma más pequeñas $[1.5-3(-4) \times 0.5-1$ $\mathrm{mm}$ vs. 3-5 x 1-1.7 mm] y pardas a pardo oscuro (vs. pardo-amarillentas a pardo-grisáceas), pecíolo de 0.7 $1 \mathrm{~mm}$ de diámetro (vs. 1-2 $\mathrm{mm}$ ) y plantas distribuidas a menor elevación [0-900 (-1500) m vs. (700-) 1200-
$1600(-2000) \mathrm{m}]$. En muchas de sus características se parece a C. centrobrasilianum, tales como su rizoma delgado y no pruinoso, pero difiere por escamas del rizoma no iridiscentes y con células no retorcidas.

Probablemente, la mayoría de ejemplares identificados como $C$. angustifolium de elevaciones inferiores a $800 \mathrm{~m}$ corresponden a la nueva especie. La clave de 
Lellinger (1988) incluye C. angustifolium en dos partes diferentes, debido probablemente a la presencia de esta otra entidad reconocida en el punto 37 (36) y a la verdadera entidad de $C$. angustifolium en el punto $50(48)$.

Etimología: El nombre de esta especie se refiere a que rizoma, pecíolo y frondas son delgados.

Campyloneurum irregulare Lellinger, Amer. Fern J. 78 (1): 14-35. 1988.

Tipo: Ecuador, Pichincha, vicinity of Quito, $3000 \mathrm{~m}$, Holdridge 1580 (US).
Lellinger (1988, 1989) menciona dos ámbitos de distribución de esta especie en Costa Rica, 800-1500 m y 2200-3300 m. En este estudio se observó que, principalmente en la parte más norteña de su distribución entre Costa Rica y Nicaragua, se encuentran plantas con rizoma no farinoso, escamas del rizoma con las células basales menos marcadamente irregulares y distribuidas a (700-) 1200-1700 m, en tanto que la entidad original tiene rizoma farinoso, células basales de las escamas del rizoma marcadamente irregulares y se encuentra a (1200-) 1700-2500 m. La variante de zonas bajas probablemente amerite consideración de variedad o de especie.

\section{Clave de las especies del complejo de Campyloneurum angustifolium en Costa Rica (ver Cuadros 1 y 2 y Figs. 2 y 3 ).}

1. Pecíolo de 1-5 (-15) cm de largo; lámina de 0.3-1.5 (-2.7) cm de ancho con nervaduras no resaltadas

1. Pecíolo de (4-) 7-24 cm de largo; lámina de (1-) 1.5-5.0 cm de ancho con nervaduras resaltadas

2. Rizoma de 1-2 (-3) mm de diámetro; escamas del rizoma con base ensanchada y ápice acuminado;pecíolo de $0.7-1.5 \mathrm{~mm}$ de diámetro

2. Rizoma de 2-5 mm de diámetro; escamas del rizoma con base no ensanchada o muy poco ensanchadas y con ápice agudo; pecíolo de 1-2 mm de diámetro

3. Rizoma de 1-3 mm de diámetro, pruinoso, frondas distantes 2-10 (-20) mm entre sí; escamas del rizoma pardo-grisáceas a grisáceas, lumen de las células transparente; 2600-3250 m

3. Rizoma de 1-2 mm de diámetro, no pruinoso, frondas distantes $1 \mathrm{~mm}$ entre sí; escamas del rizoma pardas a pardo-oscuro, lumen de las células pardo; 0-900 (-1200)

4. Escamas del rizoma con la base sésil o levemente auriculada, pardo-amarillentas a pardo-grisáceo claro, lumen translúcido

4. Escamas del rizoma peltadas o con la base fuertemente auriculada, pardas a pardo oscuro, lumen pardo

5. Base de las escamas del rizoma un poco ensanchada, células de la base de la escama irregulares

5. Base de las escamas del rizoma no ensanchada, células de la base de la escama isodiamétricas

6. Escamas del rizoma de 1-2 mm de ancho, clatradas, con base ensanchada y ápice acuminado, pardas a pardo-oscuro; lámina de (1-) $2-5 \mathrm{~cm}$ de ancho

6. Escamas del rizoma de 2-4 $\mathrm{mm}$ de ancho, no clatradas o levemente; con base no ensanchada y ápice agudo, pardo-amarillentas; lámina de 1.0-2.5 $\mathrm{cm}$ de ancho 


\section{Cuadro 1. Comparación de características morfológicas entre Campyloneurum angustifolium,} C. cooperi, $C$. gracile y $C$. irregulare.

\begin{tabular}{|c|c|c|c|c|}
\hline Característica & C. angustifolium & C. cooperi & C. gracile & C. irregulare \\
\hline Diámetro del rizoma $(\mathrm{mm})$ & $2-5$ & $2-5$ & $1.5-2.5$ & $2-5$ \\
\hline Rizoma farinoso & sí & sí o poco & no & sí (no en zonas bajas) \\
\hline $\begin{array}{l}\text { Distancia entre } \\
\text { frondas }(\mathrm{mm})\end{array}$ & $1-5$ & $1-5$ & $0-1$ & $1-5$ \\
\hline $\begin{array}{l}\text { Largo de las } \\
\text { escamas del rizoma }(\mathrm{mm})\end{array}$ & $3-5$ & $2.5-4.0$ & $1.5-3.0(-4.0)$ & $2.5-4.0$ \\
\hline $\begin{array}{l}\text { Ancho de las } \\
\text { escamas del rizoma }(\mathrm{mm})\end{array}$ & $1.0-1.7$ & $1.0-1.5$ & $0.5-1.0$ & $1.0-1.5$ \\
\hline $\begin{array}{l}\text { Color de las } \\
\text { escamas del rizoma }\end{array}$ & $\begin{array}{l}\text { pardo-amarillento a } \\
\text { pardo-grisáceo }\end{array}$ & $\begin{array}{l}\text { pardo a pardo } \\
\text { oscuro }\end{array}$ & $\begin{array}{l}\text { pardo a pardo } \\
\text { oscuro }\end{array}$ & $\begin{array}{l}\text { pardo a pardo } \\
\text { oscuro }\end{array}$ \\
\hline $\begin{array}{l}\text { Células basales de } \\
\text { las escamas del rizoma }\end{array}$ & $\begin{array}{l}\text { poligonales, } \\
\text { isodiamétricas }\end{array}$ & $\begin{array}{l}\text { poligonales, } \\
\text { isodiamétricas }\end{array}$ & $\begin{array}{l}\text { poligonales, } \\
\text { isodiamétricas }\end{array}$ & $\begin{array}{l}\text { poligonales, } \\
\text { irregulares }\end{array}$ \\
\hline $\begin{array}{l}\text { Células apicales } \\
\text { de las escamas del rizoma }\end{array}$ & $\begin{array}{l}1.5-3.0 \text { veces } \\
\text { más largas que anchas }\end{array}$ & $\begin{array}{l}1-2 \text { veces más } \\
\text { largas que anchas }\end{array}$ & $\begin{array}{l}1-2 \text { veces más } \\
\text { largas que anchas }\end{array}$ & $\begin{array}{l}1.5-3.0 \text { veces } \\
\text { más largas que anchas }\end{array}$ \\
\hline $\begin{array}{l}\text { Lumen de las } \\
\text { células de las escamas }\end{array}$ & translúcido & pardo & pardo & pardo \\
\hline $\begin{array}{l}\text { Longitud de las } \\
\text { frondas }(\mathrm{cm})\end{array}$ & $20-45(-75)$ & (9-) $15-40$ & $(10-) 18-40(-55)$ & $(10-) 20-35(-50)$ \\
\hline Largo del pecíolo $(\mathrm{cm})$ & $0.5-4.0(-9.0)$ & $0.5-4.0$ & $0.5-4.0$ & $0.5-5.0$ \\
\hline Diámetro del pecíolo $(\mathrm{mm})$ & $1-2$ & $1-2$ & $0.7-1.0$ & $1-1.5$ \\
\hline Ancho de la lámina $(\mathrm{cm})$ & $0.4-1.5(-2.0)$ & $0.3-1.0(-2.7)$ & $0.3-1.5(-2.0)$ & $0.4-1.5(-2.5)$ \\
\hline Nervaduras resaltadas & no & no & no & no \\
\hline Distribución altitudinal (m) & $(700-)$ 1200-1600 (-2000) & $(1200-) 1600-2000$ & $0-900(-1200)$ & $(700-) 1200-2500$ \\
\hline
\end{tabular}

\section{Cuadro 2. Comparación de características morfológicas entre Campyloneurum amphostenon, C. angustipaleatum y $C$. densifolium.}

\begin{tabular}{|c|c|c|c|c|}
\hline Característica & C. amphostenon 1 & C. amphostenon 2 & C. angustipaleatum & C. densifolium \\
\hline Diámetro del rizoma (mm) & $2.5-4.0(-5.0)$ & $3-5$ & $2-3$ & $3-6$ \\
\hline Rizoma farinoso & escasamente & escasamente & sí & escasamente \\
\hline $\begin{array}{l}\text { Distancia entre } \\
\text { frondas }(\mathrm{mm})\end{array}$ & $10-50(-70)$ & $2-15$ & $2-10(-20)$ & $5-20(-50)$ \\
\hline $\begin{array}{l}\text { Largo de las } \\
\text { escamas del rizoma }(\mathrm{mm})\end{array}$ & $2-5$ & $4-8$ & $3-6$ & $4-6$ \\
\hline $\begin{array}{l}\text { Ancho de las } \\
\text { escamas del rizoma (mm) }\end{array}$ & $1.0-1.5$ & $1-2$ & $1.0-1.5$ & $2-4$ \\
\hline $\begin{array}{l}\text { Color de las } \\
\text { escamas del rizoma }\end{array}$ & pardo a pardo-oscuro & $\begin{array}{l}\text { pardo-amarillento } \\
\text { a pardo-grisáceo }\end{array}$ & pardo a pardo-grisáceo & pardo-amarillento \\
\hline $\begin{array}{l}\text { Células basales de } \\
\text { las escamas del } \\
\text { rizoma }\end{array}$ & $\begin{array}{l}\text { triangulares a } \\
\text { hexagonales, } \\
\text { isodiamétricas }\end{array}$ & $\begin{array}{l}\text { triangulares a } \\
\text { hexagonales, } \\
\text { isodiamétricas }\end{array}$ & $\begin{array}{l}\text { poligonales, } \\
\text { isodiamétricas }\end{array}$ & $\begin{array}{l}\text { poligonales, } \\
\text { isodiamétricas }\end{array}$ \\
\hline $\begin{array}{l}\text { Células apicales } \\
\text { de las escamas del rizoma }\end{array}$ & $\begin{array}{l}1-2 \text { veces más } \\
\text { largas que anchas }\end{array}$ & $\begin{array}{l}1.5-3.0 \text { veces } \\
\text { más largas que anchas }\end{array}$ & $\begin{array}{l}1.5-3.0 \text { veces } \\
\text { más largas que anchas }\end{array}$ & $\begin{array}{l}1.0-1.5 \text { veces } \\
\text { más largas que anchas }\end{array}$ \\
\hline $\begin{array}{l}\text { Lumen de las } \\
\text { células de las escamas }\end{array}$ & $\begin{array}{l}\text { translúcido a } \\
\text { pardo-amarillento }\end{array}$ & translúcido & translúcido & $\begin{array}{l}\text { translúcido a } \\
\text { amarillento }\end{array}$ \\
\hline Longitud de las frondas $(\mathrm{cm})$ & (35-) 45-60 (-80) & $30-55(-65)$ & $(10-) 15-30(-45)$ & $40-70(-120)$ \\
\hline Largo del pecíolo $(\mathrm{cm})$ & $8-25$ & $4-10(-20)$ & $2-5(-15)$ & $7-24$ \\
\hline Diámetro del pecíolo $(\mathrm{mm})$ & $2-3$ & $2-3$ & $0.7-1.5$ & $2-4$ \\
\hline Ancho de la lámina (cm) & $(1.3-) 2.0-5.0$ & $1.0-3.5$ & $0.4-1.2(-1.7)$ & $1.0-2.5$ \\
\hline Nervaduras resaltadas & sí & poco o no & poco o no & sí \\
\hline Distribución altitudinal (m) & $(2400-)$ 2800-3400 & $1800-2500(-2800)$ & $2600-3250$ & $2600-3100$ \\
\hline
\end{tabular}


Lellinger, D.B. 1988. Some new species of Campyloneurum and a provisional key to the genus. Amer. Fern J. 78(1): 14-35.

Lellinger, D.B. 1989. The ferns and fern-allies from Costa Rica, Panama, and the Chocó. Part I. Pteridologia 2A. p. 255-264.

León, B. 1993. Campyloneurum. In: Tryon, R.M. \& R.G. Stolze (ed.). Pteridophyta of Perú. Part V. 18. Aspleniaceae - 21. Polypodiaceae. Fieldiana, Bot., n.s. 32: 158-173.

León, B. 1995. Campyloneurum. In: Moran, R.C. \& R. Riba (eds.). Flora Mesoamericana. Vol.1. Psilotaceae a Salviniaceae. Univ. Nac. Autónoma de México. p. 333-338.
Mickel, J.T. \& J.M. Beitel. 1988. Pteridophyte Flora of Oaxaca, México. Mem. New York Bot. Gard. 46: 96-99.

Proctor, G.R. 1985. Ferns of Jamaica, a guide to the Pteridophytes. British Museum. 631 p.

Proctor, G.R. 1989. Ferns of Puerto Rico and The Virgin Islands. Mem. New York Bot. Gard. 53: 318-322.

Rojas, A.F. 1996. Aportes a la Flora Pteridophyta Costarricense. I. Informes. Brenesia 45-46: 1-6.

Smith, A.R. 1995. Campyloneurum. In: Berry, P.E., B.K. Holst \& K. Yatskievych (eds.). Flora of the Venezuelan Guayana. Vol. 2. Pteridophytes, Spermatophytes, Acanthaceae-Araceae. Oregon, Timber Press. p. 221-225. 